\title{
ARVESON NESTS AND OPERATOR FACTORIZATION ALONG COMMUTATIVE SUBSPACE LATTICES
}

\author{
JOHN DAUGHTRY AND RONALD JOHNS
}

(Communicated by John B. Conway)

\begin{abstract}
Similar commutative subspace lattices (CSL's) are shown to be unitarily equivalent if certain sublattices (which may be taken to be nests!) are unitarily equivalent and a technical condition is satisfied. This result provides a connection between existing results for arbitrary similarities of countable CSL's and similarities of general CSL's by operators near the identity. One consequence is the generalizaton to CSL's of a theorem of David Pitts on the relationship between similarity and unitary equivalence of nests he calls "injective."
\end{abstract}

$H$ denotes a separable Hilbert space with real or complex scalars. $B(H)$ is the space of all bounded, linear operators on $H$. The word "projection" always refers to an operator of orthogonal projection. For any $A$ in $B(H), r p(A)$ denotes the projection of $H$ on the closure of the range of $A . R(A)$ is the range of $A$, and $I$ is the identity operator.

If $\Phi$ is a linear operator on $B(H)$ satisfying

(i) for all $X \in B(H)$ such that $X \geq 0, \Phi(X) \geq 0$,

(ii) $\Phi\left(X^{*}\right)=\Phi(X)^{*}$ for all $X \in B(H)$,

(iii) $\Phi$ is idempotent, and

(iv) $\Phi(X) \Phi(Y)=\Phi(X(\Phi(Y))$ for all $X$ and $Y$ in $B(H)$, then $\Phi$ is a conditional expectation from $B(H)$ onto $R(\Phi)$.

Remarks. 1. (ii) is a consequence of (i) when the scalars are complex.

2. The identity $\Phi(X) \Phi(Y)=\Phi(\Phi(X) Y)$ is easily derived from (iv) and (ii).

3. $R(\Phi)$ is the set of fixed points of $\Phi$.

4. [11; Chapter II] is a good reference for the properties of conditional expectation operators.

Let $\mathscr{C} \subset \mathscr{V} \subset B(H)$. A function $\Phi$ from $\mathscr{V}$ into itself is $\mathscr{C}$-homogeneous if $\Phi(C X)=C \Phi(X)$ and $\Phi(X C)=\Phi(X) C$ for all $C$ in $\mathscr{C}$ and $X$ in $\mathscr{V}$. (Thus we do not require any linearity or continuity.) Of course, conditional expectation operators are examples of homogeneous maps, and the significance of

Received by the editors September 6, 1988. Presented to the Great Plains Operator Theory Seminar in Indianapolis, Indiana in May, 1988.

1980 Mathematics Subject Classification (1985 Revision). Primary 47C05, 47A15, 47A68.

Key words and phrases. Commutative subspace lattice algebra, operator factorization, nest, conditional expectations on von Neumann algebras. 
the following work depends upon previously known results about the existence of conditional expectation operators of $B(H)$ onto certain subalgebras.

$\mathscr{V}$ denotes a von Neumann subalgebra of $B(H)$ throughout the following exposition. A lattice $L$ of projections in $B(H)$ is complete if the least upper bound and greatest lower bound of each subset of $L$ is in $L$. A complete lattice of projections which contains 0 and $I$ is a subspace lattice. A subspace lattice $L$ which satisfies $P Q=Q P$ for all $P$ and $Q$ in $L$ is a commutative subspace lattice (CSL). A subspace lattice which is linearly ordered is a nest.

Let $\mathscr{P}$ be any set of projections in $B(H)$, and let $T$ be an invertible element of $B(H)$. $\tau \mathscr{P}$ denotes $\{r p(T P): P \in \mathscr{P}\}$. Alg $\mathscr{P}$ denotes $\{A \in B(H): A$ leaves invariant the ranges of the elements of $\mathscr{P}\}$. The diagonal of $\mathscr{P}$, denoted $\mathscr{D}(\mathscr{P})$, is alg $\mathscr{P} \cap(\operatorname{alg} \mathscr{P})^{*}$. If $\mathscr{P} \subset \mathscr{V}$, the relative diagonal $\mathscr{D}(\mathscr{P}) \cap \mathscr{V}$ is denoted $\mathscr{D}_{\%}(\mathscr{P})$. Let $L$ be a CSL. A nest $N \subset L$ is an Arveson nest for $L$ if $N^{\prime}=L^{\prime}$. (The prime denotes the commutant.) A nest $N \subset L$ is a $T$-Arveson nest for $L$ if $N^{\prime}=L^{\prime}$ and $(\tau N)^{\prime}=(\tau L)^{\prime}$. Every CSL in a separable Hilbert space has an Arveson nest [2; p. 482]. (The maximality of the Abelian von Neumann algebra generated by the CSL, which is assumed there, is not used in this part of Arveson's proof.) In addition, we have the following

Lemma. Let $L$ be a CSL. Then $L$ contains a nest which is a simultaneous $T$-Arveson nest for every invertible operator $T$ such that $\tau L$ is commutative.

Proof. Start with a countable dense sublattice $\mathscr{S}$ of $L, \mathscr{S}=\left\{P_{1}, P_{2}, P_{3}, \ldots\right\}$. Let $L_{n}$ denote the (finite) subspace lattice generated by $\left\{P_{1}, P_{2}, \ldots, P_{n}\right\}$. Then construct a finite nest $N_{n} \subset L_{n}$ with $\left(N_{n}\right)^{\prime}=\left(L_{n}\right)^{\prime}$ and $N_{n} \subset N_{n+1}$ for $n=$ $1,2, \ldots$, as in $[2 ;$ p. 482]. For every invertible operator $T$ such that $\tau L$ is commutative, there exists a unitary operator $U_{n}$ such that $U_{n} P U_{n}^{*}=r p(T P)$ for all $P$ in $L_{n}$. (This is a reformulation of Theorem 2 of [4].) Therefore, $\left(\tau N_{n}\right)^{\prime}=\left(\tau L_{n}\right)^{\prime}$. Let $N$ denote the strong operator closure of the union of the $N_{n}{ }^{\prime}$ s. $N$ is a nest with the same commutant as $L$, and $(\tau N)^{\prime}=(\tau L)^{\prime}$.

Proposition 1. Let $T \in \mathscr{V}$ be invertible, and let $\mathscr{P} \subset \mathscr{V}$ be a commutative set of projections. Assume that $\{r p(T P): P \in \mathscr{P}\}$ is commutative, $\mathscr{M} \subset \mathscr{P}$, $\mathscr{M}^{\prime} \cap \mathscr{V}=\mathscr{P}^{\prime} \cap \mathscr{V}$, and $U$ is a unitary operator in $\mathscr{V}$ such that $U P U^{*}=$ $r p(T P)$ for all $P \in \mathscr{M}$. Let $\Phi$ be any $\mathscr{D}_{\mathscr{V}}(\mathscr{P})$-homogeneous map of $\mathscr{V}$ into $\mathscr{V}$. If $\Phi\left(T^{-1} U\right) \in \mathscr{D}_{\gamma}(\mathscr{P})$ and is one-to-one, then $R(U P) \supset R(T P)$ for all $P \in \mathscr{P}$.

Proof. $r p\left(U^{*} T P\right)=U^{*} r p(T P) U$ commutes with $r p\left(U^{*} T Q\right)=U^{*} r p(T Q) U$ for each $P \in \mathscr{P}$ and $Q \in \mathscr{M}$. Thus, by the hypothesis on $U, r p\left(U^{*} T P\right)$ belongs to $\mathscr{M}^{\prime} \cap \mathscr{V}=\mathscr{P}^{\prime} \cap \mathscr{V}$ for each $P \in \mathscr{P}$. Then

$$
\begin{aligned}
P \Phi\left(T^{-1} U\right) U^{*} T P & =P \Phi\left(T^{-1} U\right) r p\left(U^{*} T P\right) U^{*} T P \\
& =\Phi\left(P T^{-1} U r p\left(U^{*} T P\right)\right) U^{*} T P \\
& =\Phi\left(T^{-1} U r p\left(U^{*}(T P)\right) U^{*} T P=\Phi\left(T^{-1} U\right) U^{*} T P .\right.
\end{aligned}
$$


Thus, $\Phi\left(T^{-1} U\right) U^{*} T$ leaves invariant the ranges of all of the projections in $\mathscr{P}$. The facts that $\Phi\left(T^{-1} U\right)$ commutes with such projections and $\Phi\left(T^{-1} U\right)$ is oneto-one imply that $U^{*} T \in \operatorname{alg} \mathscr{P}$. Thus for all $P \in \mathscr{P}, R\left(U P U^{*}\right) \supset R(T P)$.

Corollary. Let $T \in \mathscr{V}$ be invertible, and let $\mathscr{P}$ be a commutative set of projections. Assume that $\{r p(T P): P \in \mathscr{P}\}$ is commutative, $\mathscr{M} \subset \mathscr{P}, \mathscr{M}^{\prime} \cap \mathscr{V}=$ $\mathscr{P}^{\prime} \cap \mathscr{V}$, and $U \in \mathscr{V}$ is a unitary operator such that $U P U^{*}=r p(T P)$ for all $P \in \mathscr{M}$. Assume that there exists an expectation $\Phi$ of $\mathscr{V}$ onto $\mathscr{D}_{\mathscr{V}}(\mathscr{P})$ which is multiplicative on $\mathscr{V} \cap \operatorname{alg} \mathscr{M}$. Then $R(U P) \supset R(T P)$ for all $P \in \mathscr{P}$.

Proof. The hypothesis on $r p(T P)$ implies that $U^{*} T$ and $T^{-1} U$ belong to $\mathscr{V}$ nalg $\mathscr{M}$. Then $\Phi\left(T^{-1} U\right)$ is the inverse of $\Phi\left(U^{*} T\right)$, so the desired conclusion follows from Proposition 1.

Theorem 1. Let $T \in \mathscr{V}$ be invertible and let $\mathscr{P} \subset \mathscr{V}$ be a commutative set of projections. Assume that $\tau \mathscr{P}$ is commutative, $\mathscr{M} \subset \mathscr{P}, \mathscr{M}^{\prime} \cap \mathscr{V}=\mathscr{P}^{\prime} \cap \mathscr{V}$, $(\tau \mathscr{M})^{\prime} \cap \mathscr{V}=(\tau \mathscr{P})^{\prime} \cap \mathscr{V}$, and $U$ is a unitary operator in $\mathscr{V}$ such that $U P U^{*}=$ $r p(T P)$ for all $P \in \mathscr{M}$. Let $\Phi$ be any $\mathscr{D}_{\mathscr{V}}(\mathscr{P})$-homogeneous map of $\mathscr{V}$ into $\mathscr{V}$. If $\Phi\left(U^{*} T\right)$ and $\Phi\left(T^{-1} U\right)$ belong to $\mathscr{D}_{\mathscr{V}}(\mathscr{P})$ and are one-to-one, then $R(U P)=R(T P)$ for all $P \in \mathscr{P}$.

Proof. Directly from Proposition 1, we have $R(U P) \supset R(T P)$ for all $P \in \mathscr{P}$. Now we wish to apply Proposition 1 with $\tau \mathscr{P}$ and $\tau \mathscr{M}$ in the place of $\mathscr{P}$ and $\mathscr{M}$, and $U^{*}$ and $T^{-1}$ in the place of $U$ and $T$. Define $\Psi$ by $\Psi(X)=$ $U \Phi\left(U^{*} X U\right) U^{*}$ for any $X \in \mathscr{V}$. Choose $C$ in $\mathscr{D}_{\mathscr{V}}(\tau \mathscr{P})=\mathscr{V} \cap(\tau \mathscr{P})^{\prime} . U^{*} C U$ belongs to $\left[U^{*}(\tau \mathscr{M}) U\right]^{\prime} \cap \mathscr{V}=\mathscr{M}^{\prime} \cap \mathscr{V}=\mathscr{P}^{\prime} \cap \mathscr{V}$, so $\Psi(C X)=C \Psi(X)$ and $\Psi(X C)=\Psi(X) C$. By the hypothesis on $\Phi\left(U^{*} T\right), \Psi\left(T U^{*}\right)$ is contained in $U\left(\mathscr{D}_{\mathcal{Y}}(\mathscr{P})\right) U^{*}=\left(U \mathscr{P} U^{*}\right)^{\prime} \cap \mathscr{V}=\left(U \mathscr{M} U^{*}\right)^{\prime} \cap \mathscr{V}=(\tau \mathscr{M})^{\prime} \cap \mathscr{V}=(\tau \mathscr{P})^{\prime} \cap \mathscr{V}=$

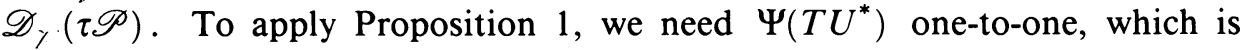
provided by the hypothesis that $\Phi\left(U^{*} T\right)$ is one-to-one. We conclude that for any $Q \in \tau \mathscr{P}, R\left(U^{*} Q\right) \supset R\left(T^{-1} Q\right)$. Thus, for any $P \in \mathscr{P}, R\left(U^{*} T P\right) \supset R(P)$, or $R(T P) \supset R(U P)$.

Definitions. A nest $N \subset \mathscr{V}$ is a subdiagonal nest in $\mathscr{V}$ if there exists an expectation $\Phi$ of $\mathscr{V}$ onto the commutant of $N$ such that $\Phi$ is multiplicative on $\mathscr{V} \cap \operatorname{alg} N$. A conditional expectation $\Phi$ on $\mathscr{V}$ is faithful if the only element $X$ in the kernel of $\Phi$ satisfying $X \geq 0$ is $X=0$. $\Phi$ is normal if it is continuous with respect to the strong operator topology.

If there exists a faithful, normal conditional expectation $\Phi$ of $\mathscr{V}$ onto the commutant of $N$, then $N$ is a subdiagonal nest with $\Phi$ the multiplicative expectation [1]. In particular, it is an elementary fact that every countable nest is subdiagonal. We now arrive at the promised generalization of Theorem 5.8 of [11] to CSL's:

Theorem 2. Let $L$ be a CSL contained in $\mathscr{V}$, a von Neumann subalgebra of $B(H)$. Suppose that there exists a faithful, normal expectation of $\mathscr{V}$ onto $\mathscr{D}_{y}(L)$. If $T \in \mathscr{V}$ is invertible, then there exists a unitary operator $U \in \mathscr{V}$ 
such that $U P U^{*}=r p(T P)$ for all $P$ in $L$ if and only if $\tau L$ is a CSL and there exists a faithful, normal expectation of $\mathscr{V}$ onto $\mathscr{D}_{\mathscr{V}}(\tau L)$.

Proof. Let $\Phi$ be the faithful normal expectation on $\mathscr{D}_{\mathscr{Y}}(L)$, and assume that $\tau L$ is a CSL and there exists a faithful, normal expectation of $\mathscr{V}$ onto $\mathscr{D}_{\mathscr{V}}(\tau L)$. Let $N$ be a $T$-Arveson nest for $L$. By Theorem 5.8 of [10], there exists a unitary operator $U \in \mathscr{V}$ such that $U P U^{*}=r p(T P)$ for all $P$ in $N . \Phi$ is multiplicative on alg $N \cap \mathscr{V}$, so $\Phi\left(U^{*} T\right)$ and $\Phi\left(T^{-1} U\right)$ are inverse to each other. We may now apply Theorem 1 with $\mathscr{P}=L$ and $\mathscr{M}=N$ to obtain $U P U^{*}=r p(T P)$ for all $P$ in $L$.

For the converse, suppose that $U$ is a unitary operator in $\mathscr{V}$ such that $U P U^{*}=r p(T P)$ for all $P$ in $L$. Then $\tau L$ is clearly a CSL, and $\Psi(X)=$ $U \Phi\left(U^{*} X U\right) U^{*}$ is the desired faithful normal expectation onto $\mathscr{D}_{\mathscr{V}}(\tau L)$.

As the hypothesis about the existence of a faithful, normal expectation is satisfied for every countable CSL, the condition that $\tau L$ is commutative is necessary and sufficient for the existence of the unitary $U$ such that $U P U^{*}=$ $r p(T P)$ for all $P$ in $L$ when $L$ is countable (cf [4; Theorem 2]).

Results such as those above are often expressed as results about operator factorization. For example, an immediate consequence of Theorem 1 (proved by letting $U=T A^{-1}$ ) is the following:

Corollary. Let $T \in \mathscr{V}$ be invertible and let $L \subset \mathscr{V}$ be a CSL such that $\tau L$ is commutative. Let $N$ be any $T$-Arveson nest for $L$ and assume that there exists $A \in \mathscr{V}$ such that $A$ is invertible and $T^{*} T=A^{*} A$ with $A$ and $A^{-1}$ in $\operatorname{alg} N$. Let $\Phi$ be any $\mathscr{D}_{Y}(L)$-homogeneous map of $\mathscr{V}$ into $\mathscr{V}$. If $\Phi(A)$ and $\Phi\left(A^{-1}\right)$ belong to $\mathscr{D}_{y}(L)$ and are one-to-one, then $A$ and $A^{-1}$ belong to $\operatorname{alg} L$.

Theorem 1 yields not only results about similarity of special CSL's, but also results about similarity (or operator factorization) of general CSL's by operators "close to the identity." We state the operator factorization form of the most important consequence:

Theorem 3 (cf. [5]). Let $L$ be a CSL contained in $\mathscr{V}$, a von Neumann subalgebra of $B(H)$. Let $S$ be an invertible, self-adjoint element of $\mathscr{V}$ with $S \geq 0$. Assume that $S=I-K$ with $K$ an element of Macaev's ideal. Then a necessary and sufficient condition that $S=A^{*} A$ with $A$ and $A^{-1}$ in $\operatorname{alg} L$ is that $\left\{r p\left(S^{1 / 2} P\right): P \in L\right\}$ is commutative.

Proof. The commutativity condition is necessary by Theorem 1 of [3].

Now assume the commutativity of $\left\{r p\left(S^{1 / 2} P\right): P \in L\right\}$. By the lemma, there exists an $S^{1 / 2}$-Arveson nest $N$ for $L$. The theory of [8] as summarized on pp. 101-102 of [7] yields a factorization $S=\left(I+V^{*}\right) D(I+V)$ with $D \in N^{\prime} \cap \mathscr{V}$ and $V$ in the Jacobson radical of alg $N$. We claim that $A=D^{1 / 2}(I+V)$ has the desired properties. There exists a conditional expectation $\Phi$ of $\mathscr{V}$ onto $L^{\prime} \cap \mathscr{V}\left(=N^{\prime} \cap \mathscr{V}\right)$ constructed by the standard technique of averaging with respect to the action of the group of unitary operators in $L^{\prime \prime}$. (See [11; p. 137], 
for example.) $\Phi(A)=D^{1 / 2}$ because $\Phi$ is $N^{\prime}$-homogeneous and the kernel of $\Phi$ contains the radical of alg $N . V$ is quasinilpotent, so $(I+V)^{-1}=I+W$ with $W$ in the radical of alg $N$. Thus $\Phi\left(A^{-1}\right)=D^{1 / 2}$. The preceding corollary now applies with $T=S^{1 / 2}$.

\section{ACKNOWLEDGMENT}

The authors are grateful to David Larson for pointing out the existence of Arveson subnests for arbitrary CSL's on separable Hilbert spaces. They thank the anonymous referee for asserting the validity of Theorem 2 for von Neumann subalgebras of $B(H)$ and pointing out that Theorem 2 for the case of $\mathscr{V}=$ $B(H)$ would have a simpler proof.

\section{REFERENCES}

1. W. B. Arveson, Analyticity in operator algebras, Amer. J. Math. 89 (1967), 578-642.

2. __ Operator algebras and invariant subspaces, Ann. of Math. 2 (1974), 433-532.

3. J. Daughtry and B. Dearden, A test for the existence of Gohberg-Krein representations in terms of multiparameter Wiener processes, J. Funct. Anal. 63 (1985), 403-411.

4. J. Daughtry, Factorizations along commutative subspace lattices, Integral Equations Operator Theory 10 (1987), 290-296.

5. Invariance of projections in the diagonal of a nest algebra, Proc. Amer. Math. Soc. 102 (1988), 117-120.

6. Conditional expectations and invariant subspaces, in Contributions to Operator Theory and its Applications, vol. 35, Birkhäuser, Boston, 1988, 23-36.

7. A. Feintuch and R. Saeks, System theory: A Hilbert space approach, Academic Press, New York, 1982.

8. I. Gohberg and M. G. Krein, Theory and application of Volterra operators in Hilbert space, A. M. S. Transl. of Math. Mono., vol. 24, 1970.

9. D. R. Larson, Nest algebras and similarity transformation, Ann. of Math. 121 (1985), 409-427.

10. David R. Pitts, Factorization problems for nests: factorization methods and characterizations of the universal factorization property, J. Funct. Anal. 79 (1988), 57-90.

11. S. Strătilă. Modular theory in operator algebras, Abacus Press, Kent, England, 1981. 27858

Department of Mathematics, East Carolina University, Greenville, North Carolina 